\title{
THE PONTRYAGIN MAXIMUM PRINCIPLE FROM DYNAMIC PROGRAMMING AND VISCOSITY SOLUTIONS TO FIRST-ORDER PARTIAL DIFFERENTIAL EQUATIONS
}

\author{
EMMANUEL NICHOLAS BARRON ${ }^{1}$ AND ROBERT JENSEN $^{2}$
}

\begin{abstract}
We prove the Pontryagin Maximum Principle for the Lagrange problem of optimal control using the fact that the value function of the problem is the viscosity solution of the associated Hamilton-Jaccbi-Bellman equation. The proof here makes rigorous the formal proof of Pontryagin's principle known for at least three decades.
\end{abstract}

The Pontryagin Maximum Principle (PMP) is the major tool in optimal control theory used in deriving an optimal control. The PMP is a set of necessary conditions which an optimal control must satisfy. Because of its importance theoretically and for applications, it has several proofs in the literature, beginning with the original proof by Pontryagin, et al. [7] in the early 1950s. We refer the reader to the recent book by Cesari [2] for two proofs of the PMP and the references cited there for other proofs and related results. In general, these proofs are technically difficult and very long. However, for many years a formal derivation of the PMP using dynamic programming and the associated Hamilton-Jacobi-Bellman (HJB) equation has been known. See for example, Berkovitz [1], Lee and Markus [5], or the end of $\S 1$ of this paper for the formal derivation. While the formal proof is very short and simple, it is strictly formal in that it requires the value function for the control problem to be $C^{2}$. Of course, the value function is usually not $C^{2}$, but it is a solution of the equation HJB (see below), a first-order, nonlinear partial differential equation.

In recent years, commencing with the pioneering paper of Crandall and Lions [3], a notion of global solution to a first-order nonlinear pde has been developed which leads to global existence, uniqueness, and stability. This solution is called the viscosity solution. (Refer to $\S 1$ for definitions.) For optimal control theory it is important that the value function is the viscosity solution of the equation HJB.

One of the most important aspects of the theory of viscosity solutions is that it provides a sort of maximum principle (in the sense of partial differential equations) for first order pde's. In fact, it is part of the definition. (This definition is given in $\S 1$ and is introduced in the paper by Crandall, Evans, and Lions [4].) It is this aspect of viscosity theory that we use in this paper to prove the PMP by the dynamic programming approach. We use only the fact that the value function

Received by the editors November $5,1985$.

1980 Mathematics Subject Classification (1985 Revision). Primary 49C20, 49B10.

Key words and phrases. Pontryagin Maximum Principle, optimal control, viscosity solutions, first-order partial differential equations, dynamic programming.

1 Partially supported by a grant from the AFOSR.

2 Alfred P. Sloan Foundation Fellow. Also partially supported by a grant from the NSF. 
is the viscosity solution of the equation HJB (actually we need only that it is a subsolution) and a differentiability property of the value function along trajectories to prove the PMP for the Lagrange problem under the standard hypotheses for the PMP. Other than these facts, the remaining results of viscosity solutions are not used.

The proof given in this paper thus makes rigorous the formal proof using dynamic programming and Bellman's equation. It retains the simplicity of the formal proof and uses only elementary results.

1. Preliminaries. We will consider the following optimal control problem to illustrate the use of viscosity techniques in optimal control. The dynamics are given by the system of ordinary differential equations

$$
\begin{gathered}
d x / d t=f(t, x(t), u(t)) \quad \text { if } 0 \leq s<t \leq T, \\
x(s)=y, \quad y \text { in } R^{n} .
\end{gathered}
$$

The control functions $u:[s, T] \rightarrow U$ are chosen from the class $K[s, T]$ of measurable functions with values almost everywhere in the fixed set $U \subset R^{p}, p$ a positive integer. The controls are chosen to maximize the payoff functional

$$
P(u)=g(x(T))+\int_{s}^{T} h(r, x(r), u(r)) d r .
$$

The given functions $f, g$, and $h$ are assumed throughout this paper to satisfy the condition (which can be weakened):

$f:[0, T] \times R^{n} \times U \rightarrow R^{n}$ is bounded and continuous in all arguments and $\partial f(t, x, u) / \partial x$ is continuous;

(A) $\quad h:[0, T] \times R^{n} \times U \rightarrow R^{1}$ is bounded and continuous in all arguments and $\partial h(t, x, u) / \partial x$ is continuous;

$g: R^{n} \rightarrow R^{1}$ is continuously differentiable and bounded.

If we look at this problem as depending on the initial conditions, then we define the value function

$$
V:[0, T] \times R^{n} \rightarrow R^{1}, \quad V(s, y)=\sup \left\{P_{s, y}(u) ; u \in K[s, T]\right\} .
$$

We also denote the trajectory corresponding to a control $u$ by $x(t ; s, y)$ if $x(s ; s, y)$ $=y$ is the initial state.

It is well known that, under condition (A), $V$ is uniformly Lipschitz in $(s, y)$ and hence has a total derivative almost everywhere. At each point $(s, y)$ of differentiability, $V$ satisfies the Hamilton-Jacobi-Bellman equation

$$
V_{s}(s, y)+\max _{u \in U}\left\{f(s, y, u) \cdot V_{y}(s, y)+h(s, y, u)\right\}=0
$$

with terminal condition

$$
V(T, y)=g(y), \quad \forall y \text { in } R^{n}
$$

where $V_{s}$ and $V_{y}=\left(V_{y_{1}}, \ldots, V_{y_{n}}\right)$ denote the partials of $V$.

It is also true, and easily proved (see [6]) that $V$ is the unique viscosity solution of (HJB)-(TC). For the reader's convenience we recall the definition here of a viscosity solution of a first order Hamilton-Jacobi equation. 
Definition. Given $H:[0, T] \times R^{n} \times R^{n} \rightarrow R^{1}$ continuous and $g: R^{n} \rightarrow R^{1}$ bounded and uniformly continuous, a bounded, uniformly continuous function $u:[0, T] \times R^{n} \rightarrow R^{1}$ is a viscosity solution of

$$
\begin{gathered}
u_{t}+H\left(t, x, u_{x}\right)=0 \quad \text { in }(0, T) \times R^{n}, \\
u(T, x)=g(x) \quad \text { in } R^{n}
\end{gathered}
$$

if (1.5) holds and if for each $\phi$ in $C^{1}\left((0, T) \times R^{n}\right)$

(a) if $u-\phi$ attains a local maximum at $\left(t_{0}, x_{0}\right) \in(0, T) \times R^{n}$, then

$$
\phi_{t}\left(t_{0}, x_{0}\right)+H\left(t_{0}, x_{0}, \phi_{x}\left(t_{0}, x_{0}\right)\right) \geq 0
$$

and

(b) if $u-\phi$ attains a local minimum at $\left(t_{0}, x_{0}\right) \in(0, T) \times R^{n}$, then

$$
\phi_{t}\left(t_{0}, x_{0}\right)+H\left(t_{0}, x_{0}, \phi_{x}\left(t_{0}, x_{0}\right)\right) \leq 0 .
$$

The function $\phi$ can be assumed to be infinitely differentiable. All that is necessary is differentiability at the extrema. For the basic results concerning viscosity solution see [4].

Our interest here is to derive the Pontryagin Maximum Principle for the optimal control problem using the idea of viscosity solution. We now recall the formal proof of Pontryagin's principle for convenience.

Suppose $u^{*}$ is optimal and $x^{*}$ is the corresponding optimal trajectory. Then

$$
V\left(t, x^{*}(t)\right)=g\left(x^{*}(T)\right)+\int_{t}^{T} h\left(r, x^{*}(r), u^{*}(r)\right) d r
$$

satisfies $V\left(T, x^{*}(T)\right)=g\left(x^{*}(T)\right)$ and, since $V_{t}+\max _{u}\left\{V_{x} \cdot f+h\right\}=0$,

$$
V_{t}\left(t, x^{*}(t)\right)+V_{x}\left(t, x^{*}(t)\right) f\left(t, x^{*}(t), u^{*}(t)\right)+h\left(t, x^{*}(t), u^{*}(t)\right)=0 .
$$

Differentiate with respect to $x$ evaluated along $\left(t, x^{*}(t)\right)$ and let $p(t)=V_{x}\left(t, x^{*}(t)\right)$. Noticing that $d p / d t=V_{x t}+V_{x x} f$, we get

$$
d p / d t=-p(t) f_{x}\left(t, x^{*}(t), u^{*}(t)\right)-h_{x}\left(t, x^{*}(t), u^{*}(t)\right)
$$

and

$$
p(T)=g^{\prime}\left(x^{*}(T)\right) .
$$

Hence, if $u^{*}$ is optimal, we have $u^{*}$ satisfying

$$
\begin{aligned}
\max _{u \text { in } U} & \left\{p(t) f\left(t, x^{*}(t), u\right)+h\left(t, x^{*}(t), u\right)\right\} \\
= & p(t) f\left(t, x^{*}(t), u^{*}(t)\right)+h\left(t, x^{*}(t), u^{*}(t)\right),
\end{aligned}
$$

and $p$ is given by (1.6)-(1.7). The utility of (1.6) is that the Bellman equation does not have to be solved to determine $u^{*}$.

2. The Pontryagin principle by viscosity methods. Assume that $u^{*}:[s, T]$ $\rightarrow U$ is an optimal control for (1.1)-(1.3) with initial state $(s, y)$ fixed, and suppose that $x^{*}=x^{*}(t ; s, y)$ is the associated optimal trajectory. The control $u^{*}$ will be fixed for the remainder of this paper. Thus, we define

$$
F(t, x)=f\left(t, x, u^{*}(t)\right) \quad \text { and } \quad H(t, x)=h\left(t, x ; u^{*}(t)\right) .
$$


Then $F:[s, T] \times R^{n} \rightarrow R^{n}, H:[s, T] \times R^{n} \rightarrow R^{1}$ are measurable in $t$, are bounded, and have continuous partials in $x$ uniformly in $t$.

Define the function $w:[s, T] \times R^{n} \rightarrow R^{1}$ by

$$
w(\tau, \xi)=g(x(T ; \tau, \xi))+\int_{\tau}^{T} H(r, x(r ; \tau, \xi)) d r,
$$

where

$$
\begin{gathered}
d x / d t=F(t, x(t ; \tau, \xi)), \quad s \leq \tau<t \leq T, \\
x(\tau ; \tau, \xi)=\xi \quad \text { in } R^{n} .
\end{gathered}
$$

PROPOSITION 1. (i) $w(s, y)=V(s, y)=P\left(u^{*}\right)$.

(ii) $w\left(t, x^{*}(t ; s, y)\right)=V\left(t, x^{*}(t ; s, y)\right)$ if $s \leq t \leq T$.

ProOF. (i) is by definition of $u^{*}$ and $V$. Part (ii) is the statement of Bellman's principle of optimality. Namely, that if $\left(u^{*}, x^{*}\right)$ is optimal for the initial point $(s, y)$, then it remains optimal starting from any point along the optimal trajectory.

The proof of Pontryagin's principle given below depends in an essential way on the differentiability properties of $w$ contained in the following.

LEMmA 2. Let condition (A) hold. Then $w$ is differentiable along any trajectory $(t, x(t ; \tau, \xi))$ for almost every $s \leq \tau \leq t \leq T$, where

$$
\begin{gathered}
d x / d t=F(t, x(t ; \tau, \xi)), \quad s \leq \tau \leq t \leq T, \\
x(\tau ; \tau, \xi)=\xi \quad \text { in } R^{n} .
\end{gathered}
$$

Furthermore, at a point of differentiability

$$
D w(t, x(t ; \tau, \xi))=\left(d w(t, x(t)) / d t-F(t, x(t)) w_{x}(t, x(t)), w_{x}(t, x(t))\right) .
$$

PROOF. We first claim that $w$ has the following properties:

(a) $w$ is differentiable almost everywhere in $[s, T] \times R^{n}$;

(b) the functions $\phi(t) \equiv d w(t, x(t)) / d t$ and $\phi_{x}(t) \equiv d w_{x}(t, x(t)) / d t$ exist as bounded measurable functions.

The claim is easily proved using the definition of $w$, condition (A), and standard results from the theory of ordinary differential equations. In particular, that $\phi_{x}(t)$ is bounded measurable follows from condition (A) and the fact that $\gamma(t)=\partial x(t ; \tau, \xi) / \partial \xi$ exists and is the unique absolutely continuous solution of the linear system

$$
d \gamma / d t=F_{x}(t, x(t ; \tau, \xi)) \gamma(t), \quad \gamma(\tau)=1 .
$$

Now, let $t$ be a point for which $d w(t, x(t)) / d t, d w_{x}(t, x(t)) / d t$, and $d x(t) / d t$ exist. The set of such $t$ 's is of full measure. Let $\varepsilon, \delta>0$. We have

$$
\begin{aligned}
& I \equiv w(t+\varepsilon, x(t)+\delta)-w(t, x(t)) \\
&=w(t+\varepsilon, x(t+\varepsilon))-w(t, x(t)) \\
&+w(t+\varepsilon, x(t+\varepsilon)+\delta+x(t)-x(t+\varepsilon))-w(t+\varepsilon, x(t+\varepsilon)) \\
& \equiv I_{1}+I_{2} .
\end{aligned}
$$

For $I_{1}$, since $d w(t, x(t)) / d t$ exists, we get

$$
I_{1}=\phi(t) \varepsilon+o(\varepsilon) .
$$


For $I_{2}$, we obtain

$$
\begin{aligned}
I_{2}= & w(t+\varepsilon, x(t+\varepsilon)+\delta+x(t)-x(t+\varepsilon))-w(t+\varepsilon, x(t+\varepsilon)) \\
= & w_{x}(t+\varepsilon, x(t+\varepsilon))[\delta+x(t)-x(t+\varepsilon)]+o(\delta+x(t)-x(t+\varepsilon)) \\
& \quad \text { (since } \phi_{x}(\cdot) \text { exists everywhere) } \\
= & w_{x}(t+\varepsilon, x(t+\varepsilon))[\delta+x(t)-x(t+\varepsilon)]+o(\delta, \varepsilon) \\
= & {\left[w_{x}(t+\varepsilon, x(t+\varepsilon))-w_{x}(t, x(t))\right][\delta+x(t)-x(t+\varepsilon)] } \\
& +w_{x}(t, x(t))[\delta+x(t)-x(t+\varepsilon)]+o(\delta, \varepsilon) \\
= & {\left[\phi_{x}(t) \varepsilon+o(\varepsilon)+\phi_{x}(t)\right][\delta+x(t)-x(t+\varepsilon)]+o(\delta, \varepsilon) } \\
= & {\left[\phi_{x}(t) \varepsilon+o(\varepsilon)+\phi_{x}(t)\right][\delta-F(t, x(t)) \varepsilon+o(\varepsilon)]+o(\delta, \varepsilon) } \\
= & \phi_{x}(t) \delta-\phi_{x}(t) F(t, x(t)) \varepsilon+o(\delta, \varepsilon) .
\end{aligned}
$$

Hence

$$
I=I_{1}+I_{2}=\left(\phi(t)-\phi_{x}(t) F(t, x(t))\right) \varepsilon+\phi_{x}(t) \delta+o(\delta, \varepsilon),
$$

and the lemma is proved.

REMARK. The essential point of the lemma is that $w$ is differentiable at almost every point of an arbitrary trajectory-a set of measure zero.

REMARK. Notice that

$$
w_{x}(\tau, \xi)=g^{\prime}(x(T ; \tau, \xi)) \partial x(T ; \tau, \xi) / \partial \xi+\int_{\tau}^{T} H_{x}(r, x(r ; \tau, \xi)) \partial x(r ; \tau, \xi) / \partial \xi d r .
$$

Corollary 3. At almost every $(t, x(t ; \tau, \xi)), s \leq \tau \leq t \leq T$, we have

$$
w_{t}(t, x(t ; \tau, \xi))+f\left(t, x(t ; \tau, \xi), u^{*}(t)\right) w_{x}(t, x(t ; \tau, \xi))+h\left(t, x(t ; \tau, \xi), u^{*}(t)\right)=0 .
$$

Also $w(T, x(T ; \tau, \xi))=g(x(T ; \tau, \xi))$.

PROOF. By definition of $w$,

$$
d w(t, x(t ; \tau, \xi)) / d t=-H(t, x(t ; \tau, \xi)) .
$$

But, by the chain rule,

$$
d w(t, x(t ; \tau, \xi)) / d t=w_{t}(t, x(t ; \tau, \xi))+w_{x}(t, x(t ; \tau, \xi)) F(t, x(t ; \tau, \xi)),
$$

and the corollary follows.

Now, we have that $u^{*}$ is optimal for the initial state $(s, y)$, and $x^{*}=x^{*}(t ; s, y)$, $s \leq t \leq T$ is the optimal trajectory. Then

$$
\begin{gathered}
w(T, y)=g(y), \quad w(s, y)=V(s, y), \\
w\left(t, x^{*}(t ; s, y)=V\left(t, x^{*}(t ; s, y)\right) \quad \text { if } s \leq t \leq T,\right.
\end{gathered}
$$

and

$$
V(\tau, \xi) \geq w(\tau, \xi) \quad \text { if }(\tau, \xi) \in[s, T] \times R^{n}
$$

since $V$ provides the largest payoff for each initial state.

Therefore, $V-w$ attains a minimum (of 0 ) along the entire optimal trajectory $\left(t, x^{*}(t ; s, y)\right), s \leq t \leq T$. Since $V$ is the (unique) viscosity solution of (HJB)(TC) and since $w$ is differentiable at almost every $\left(t, x^{*}(t ; s, y)\right)$, we may apply the 
definition of viscosity solution to conclude that

$$
w_{t}\left(t, x^{*}(t)\right)+\max _{u \in U}\left\{w_{x}\left(t, x^{*}(t)\right) f\left(t, x^{*}(t), u\right)+h\left(t, x^{*}(t), u\right)\right\} \leq 0
$$

for a.e $s \leq t \leq T$.

By Corollary 3 we have instead that for a.e. $s \leq t \leq T$,

$$
w_{t}\left(t, x^{*}(t)\right)+\max _{u \in U}\left\{w_{x}\left(t, x^{*}(t)\right) f\left(t, x^{*}(t), u\right)+h\left(t, x^{*}(t), u\right)\right\} \geq 0 .
$$

Hence the left-hand side must equal 0 for a.e. $s \leq t \leq T$. Combining the resulting equation with that in Corollary 3 we have

$$
\begin{aligned}
& \max _{u \text { in } U}\left\{w_{x}\left(t, x^{*}(t)\right) f\left(t, x^{*}(t), u\right)+h\left(t, x^{*}(t), u\right)\right\} \\
& \quad=w_{x}\left(t, x^{*}(t)\right) f\left(t, x^{*}(t), u^{*}(t)\right)+h\left(t, x^{*}(t), u^{*}(t)\right)
\end{aligned}
$$

for a.e. $s \leq t \leq T$.

We have proved

THEOREM 4. Let condition (A) hold and let $u^{*}$ be an optimal control on $[s, T]$ for the initial state $(s, y)$. Let $x^{*}(t ; s, y)$ be the corresponding optimal trajectory. Then $u^{*}$ satisfies

$$
\begin{aligned}
& \max _{u \text { in } U}\left\{w_{x}\left(t, x^{*}(t)\right) f\left(t, x^{*}(t), u\right)+h\left(t, x^{*}(t), u\right)\right\} \\
& \quad=w_{x}\left(t, x^{*}(t)\right) f\left(t, x^{*}(t), u^{*}(t)\right)+h\left(t, x^{*}(t), u^{*}(t)\right)
\end{aligned}
$$

almost everywhere in $[s, T]$, with $w_{x}\left(t, x^{*}(t)\right)$ absolutely continuous on $[s, T]$, $w_{x}\left(t, x^{*}(t)\right)=g^{\prime}\left(x^{*}(T)\right) \partial x^{*}(T ; s, y) / \partial y+\int_{t}^{T} h_{x}\left(r, x^{*}(r), u^{*}(r)\right) \partial x^{*}(r ; s, y) / \partial y d r$ and where

$$
w\left(t, x^{*}(t)\right)=g\left(x^{*}(T)\right)+\int_{t}^{T} h\left(r, x^{*}(r), u^{*}(r)\right) d r .
$$

The classical adjoint or costate equations can now be derived rigorously by approximation of $f, h$.

For each $\varepsilon>0$, let $H_{\varepsilon}(\tau, \xi)$ and $F_{\varepsilon}(\tau, \xi)$ be $C^{1}\left([s, T] \times R^{n}\right)$ satisfying the properties that

$$
\begin{gathered}
\left\|G_{\varepsilon}(\cdot, \xi)-G(\cdot, \xi)\right\|_{L^{1}[s, T]} \rightarrow 0, \\
\left\|K_{\varepsilon}(\tau, \cdot)-K(\tau, \cdot)\right\|_{L^{\infty}\left(R^{n}\right)} \rightarrow 0 \quad \text { as } \varepsilon \rightarrow 0
\end{gathered}
$$

for $G=F$ and $H, K=F, H, F_{x}, H_{x}$. Then

$$
w_{\varepsilon}(\tau, \xi) \equiv g\left(x_{\varepsilon}(T ; \tau, \xi)\right)+\int_{\tau}^{T} H_{\varepsilon}\left(s, x_{\varepsilon}(s ; \tau, \xi)\right) d s
$$

with

$$
d x_{\varepsilon} / d t=F_{\varepsilon}\left(t, x_{\varepsilon}(t ; \tau, \xi)\right), \quad x_{\varepsilon}(\tau ; \tau, \xi)=\xi
$$

is the unique $C^{1}$ solution of the linear problem

$$
w_{\varepsilon \tau}(\tau, \xi)+w_{\varepsilon \xi}(\tau, \xi) F_{\varepsilon}(\tau, \xi)+H_{\varepsilon}(\tau, \xi)=0 \quad \text { in }[s, T) \times R^{n}
$$




$$
w_{\varepsilon}(T, \xi)=g(\xi) \quad \text { in } R^{n}
$$

Furthermore, $\left\|w_{\varepsilon}-w\right\|$ and $\left\|w_{\varepsilon \xi}-w_{\xi}\right\| \rightarrow 0$ in $L^{\infty}\left([s, T] \times R^{n}\right)$.

Now we apply the classical theory of characteristics to this problem since $w$ is smooth, to derive that the characteristic $p_{\varepsilon}(t)=w_{\varepsilon x}(t, x(t ; \tau, \xi))$ satisfies

$$
\begin{gathered}
d p_{\varepsilon} / d t=-p_{\varepsilon}(t) F_{\varepsilon x}\left(t, x_{\varepsilon}(t ; \tau, \xi)\right)-H_{\varepsilon x}\left(t, x_{\varepsilon}(t ; \tau, \xi)\right) \quad \text { if } s \leq \tau \leq t \leq T, \\
p_{\varepsilon}(T)=g^{\prime}\left(x_{\varepsilon}(T ; \tau, \xi)\right) .
\end{gathered}
$$

Taking the limit as $\varepsilon \rightarrow 0$ of both sides of the above equations, we have

$$
\begin{gathered}
d p / d t=-p(t) F_{x}(t, x(t ; \tau, \xi))-H_{x}(t, x(t ; \tau, \xi)), \\
p(T)=g^{\prime}(x(T ; \tau, \xi)),
\end{gathered}
$$

with $p(t)=w_{x}(t, x(t ; \tau, \xi))$. That is, $w_{x}(t, x(t ; \tau, \xi))$ is the solution of the classical adjoint equation.

\section{BIBLIOGRAPHY}

1. L. D. Berkovitz, Optimal control theory, Applied Mathematical Sciences No. 12, SpringerVerlag, Berlin and New York, 1974.

2. L. Cesari, Optimization theory and applications: Problems with ordinary differential equations, Applications of Mathematics No. 17, Springer-Verlag, Berlin and New York, 1983.

3. M. G. Crandall and P. L. Lions, Viscosity solutions of Hamilton-Jacobi equations, Trans. Amer. Math. Soc. 277 (1983), 1-42.

4. M. G. Crandall, L. C. Evans, and P. L. Lions, Some properties of viscosity solutions of Hamilton-Jacobi equations, Trans. Amer. Math. Soc. 282 (1984), 487-502.

5. E. B. Lee and L. Markus, Foundations of optimal control theory, Wiley, New York, 1967.

6. P. L. Lions, Generalized solutions of Hamilton-Jacobi equations, Pitman, London, 1982.

7. L. S. Pontryagin, et al., The mathematical theory of optimal processes, Interscience, New York, 1962.

Department of Mathematical Sciences, loyola University, Chicago, IlliNOIS 60626 